Traditions, translations and transfers, direct exchanges and reinterpretations of $\mathrm{HfC}$ Bauhaus in Chile

\section{| RESUMEN |}

La escuela superior de Diseño Bauhaus fundada en la ciudad de Weimar en 1919, y clausurada en Berlín en 1933, ha sido un referente fundamental para todo el desarrollo de las artes, el diseño y la arquitectura del siglo veinte. Sin embargo no constituye un cuerpo homogéneo, y como su propio fundador Walter Gropius insistió, uno de los objetivos era que cada estudiante fuera formulando su autonomía a partir del contraste de las diferentes tendencias que era posible observar en ella. Durante el siglo veinte en Sudamérica se intentó por diferentes caminos construir un modelo de modernidad alternativa, que modificara el modo de vida de acuerdo a ideas como las que defendió esta institución. A continuación se expone un esquema general de estos procesos para el caso chileno en la relación de las disciplinas proyectuales.

\section{| ABSTRACT |}

The Bauhaus design school, founded in the city of Weimar in 1919 and closed in Berlin in 1933 has been an essential reference for the entire development of the arts, design and architecture of the twentieth century. But the Bauhaus is not a homogeneous body, and as its founder Walter Gropius insisted, one of the aims was student autonomy starting from the contrast of different trends observed in them. During the twentieth century in South America attempts have been made to build an alternative model of modernity to modify the way of life according to ideas of those who defended the Bauhaus. This paper proposes a general outline of these processes for the Chilean case related to projects within the discipline.

DAVID MAULEN DE LOS REYES*

\title{
Tradiciones, traducciones y transferencias: intercambios directos y reinterpretaciones de la HfG Bauhaus en Chile
}

"(...) la Bauhaus no es un estilo". Walter Gropius

\section{EL "PRIMER AÑO DE PRUEBA" DE LA NUEVA ESCUELA DE ARTE DE 1928}

La Escuela Superior de Diseño Bauhaus (Hochschule für Gestaltung o HfG) surgió en el contexto de los cambios radicales, sociales y culturales, que se produjeron tras la Primera Guerra Mundial, unificando la intención por construir un nuevo tiempo y que conocemos hoy genéricamente como vanguardia. Esta situación también definía a su manera un espíritu equivalente en toda América Latina, identificando a la educación como una de las principales herramientas de transformación para lograr estos cambios. En ese contexto, la metodología didáctica de la Nueva Escuela de Arte de 1928 en Chile se insertó en la reforma de la "Escuela Activa", gestándose desde 1920 y que se concreta en todo el sistema educativo, desde la educación primaria a la principal Escuela de Arte del Estado. Este fue uno de los momentos de institucionalización de la vanguardia más profundo que tuvo la enseñanza artística en el país (Maulen, 2005). Su Director Carlos Isamitt había investigado entre los años 1924 y 1926 más de trescientos planes de estudio en Europa, e implementó la metodología de "Primer año de Prueba", equivalente al "Curso de Inicio" o "Vorkurs" creado por Johannes Itten en la Bauhaus de Weimar, con ideas de Friedrich Fröebel, John Dewey y los teóricos de la Gestalt. En Chile se enseñaron sus ejercicios de abstracción y color según la metodología de "círculo cromático" y la percepción de formas geométricas elementales, como lo planteaban Wolfgang Khöler y Kurt Koffka para sus conceptos de la "Gestalt" (traducible como "configuración" o "diseño"), el principal soporte teórico de respuestas psicológicas y biológicas a fenómenos físicos utilizado en la enseñanza de la Bauhaus.

* David F. Maulen de los Reyes, desde 1999 investigador de interfaces entre arte, ciencia, tecnología y sociedad en Chile y Sudamérica. Lic. Arte U. Ch. Diploma en Estudios Culturales, Mg. Comunicación UACh. Diseñador de visualización de datos para trayectorias del edificio UNCTAD III en centro cultural BiblioGam, y mapa cualitativo de relaciones entre arquitectura y performance para SCL21 10. Curador de la III Bienal de Arte: Situación del arte contemporáneo chileno, MNBA. Colaborador de documenta de Kassel XII: Żes la modernidad nuestra antiguedad? Coordinador de contenidos del ciclo Bauhaus en Chile, Goethe Institut, MNBA, Fundación Bauhaus Dessau, 2014 (contacto: constructista@gmail.com).

** Este trabajo a contado con el apoyo del Goethe Institut Chile como actividad complementaria a la exposición Bauhaus Films, MNBA 2013 y 2014 . Agradecimientos especiales a Lisa Fluekiger, Maitén Arns y Volker Redder. 
1. Ilustraciones de la metodología del "primer año de prueba" en la Nueva Escuela de Arte de 1928, en Chile, realizadas por E. Oteíza Mardones para la exposición de fin de año. Indicación de la percepción de formas geométricas elementales como lo explicaban las teorías de la Gestalt, utilizadas en la Bauhaus. Fuente: Archivo Dionis Isamitt Danitz.

2. Ilustraciones de la metodología del "primer año de prueba" en la Nueva Escuela de Arte de 1928, en Chile, realizadas por E. Oteíza Mardones para la exposición de fin de año. "Circulo Cromático", muy similar al utilizado por Johannes Itten en el "primer año de inicio" o "Vorkurs" de la Bauhaus de Weimar. Fuente: Archivo Dionis Isamitt Danitz.

3. Explicación de la Metodología de abstracción a partir de una flor de cardo del "Primer año de Prueba" de la Nueva Escuela de Arte de 1928, en Chile realizados por Beatriz Danitz. Existe una similitud con la metodología de Johannes Itten en el "Curso de Inicio" o "Vorkurs" que él creó en la Bauhaus de Weimar.

Siguiendo esta línea, una interpretación más compleja de lo que fue la Bauhaus se vincula con el planteamiento de Walter Gropius respecto a contraponer tendencias para fomentar la autonomía del estudiante a través de su propio proceso reflexivo de síntesis ${ }^{[1]}$. Extremando esta dinámica, uno de los primeros docentes de la Bauhaus, Gerhard Marcks, es muy gráfico al buscar la síntesis entre la tradición cerámica cercana a Weimar, para lograr soluciones respecto a la tecnología disponible en los años veinte. Este tipo de búsquedas está más emparentado con las motivaciones de una escuela como la reinterpretación que Carlos Isamitt hacía en Chile de estas ideas. Por ejemplo, Gerhard Marcks, conocido como escultor, alrededor de 1926 desarrolló implementos de cocina transparentes e incluso una cafetera como las que hoy se usan de manera cotidiana, es decir, practicó una síntesis entre tradiciones de arte popular con las del avance de la tecnología, con una orientación social funcionalista (Wingler, 1975).

Para la construcción de esta nueva época, tanto en Europa Central como en Sudamérica, era necesario partir de cero estudiando los conceptos básicos de realidad: la forma, el tiempo y el espacio. Los resultados de la reforma chilena se visibilizaron en diversas publicaciones, como la RevISTA DE ARTE de la misma Escuela, con trabajos de los alumnos Marcial Lema, Gabriela Rivadeneira y Ventura Galván, y en la exposición realizada a finales de ese mismo año en la que se vio el proceso de enseñanza que incluía el modelado en bloque de composiciones geométricas, similares a las de George Vantongerloo y Johannes Itten, o el desarrollo de diagramación y tipografía funcionalista. Esta reforma de la enseñanza artística, interrumpida en enero de 1929, vinculó a la vez planteamientos equivalentes de Alemania, Italia, Bélgica, Francia, Austria,

Testimonio de Walter Gropius en exposición "Bauhaus Films", Museo Nacional de Bellas Artes, Santiago de Chile, Fundación Bauhaus Dessau, Goethe Institut Santiago, enero a marzo 2014, curatoría de Christian Hiller.
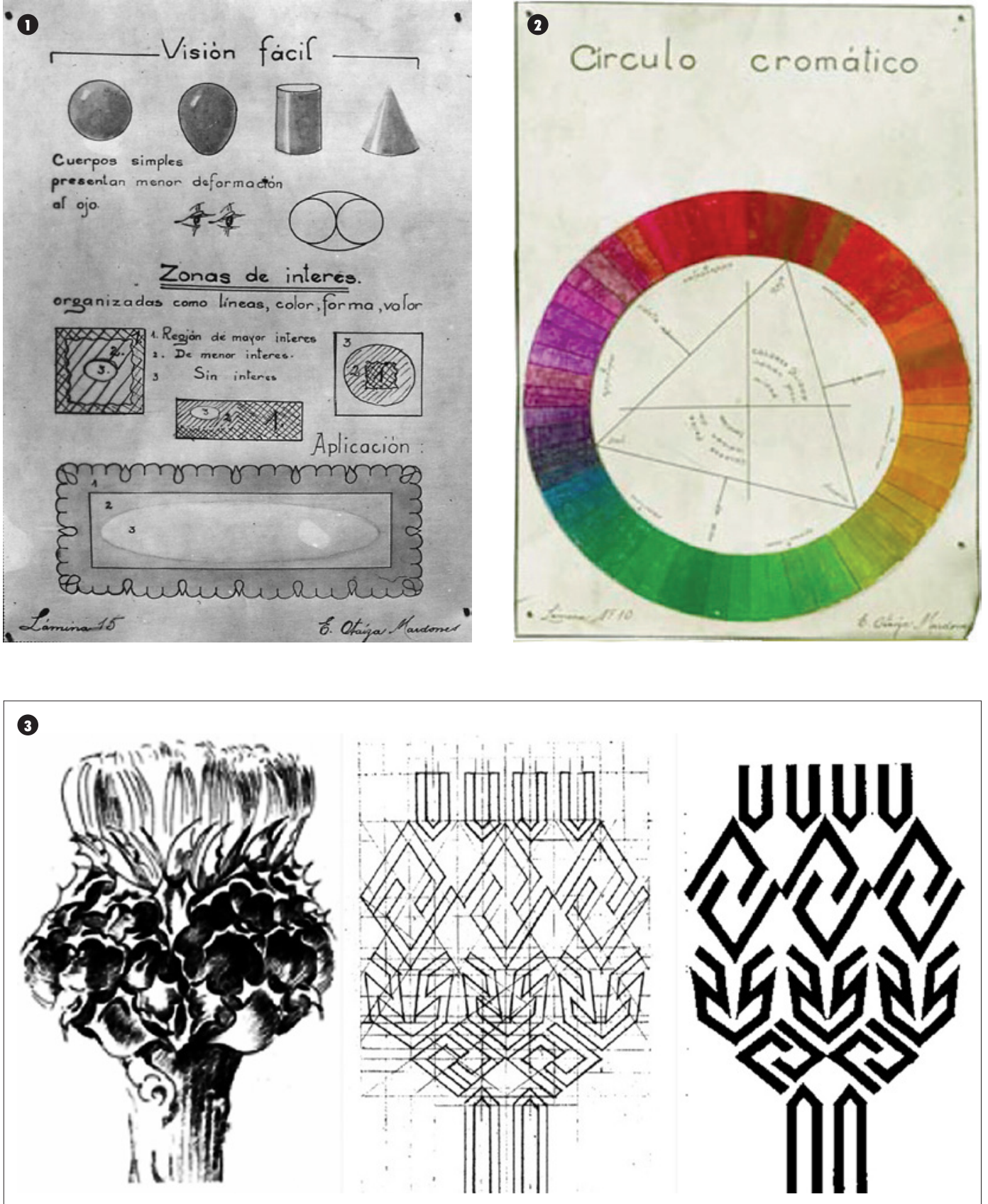

Polonia, Unión Soviética, Holanda, Estados Unidos, México, Perú y Argentina, respecto a la construcción de conocimiento a partir de reconocer las variables culturales propias, pero de manera constructiva, no ilustrativa (Jedlicki, 2005)
Ideas similares fueron introducidas por Lucio Costa en la Escuela Nacional de Bellas Artes de São Paulo en 1932. Así como la experiencia chilena de 1928 también se utilizó conceptualmente en la creación del Instituto Pedagógico de Caracas, Venezuela. 
4. Revista de Arte de la Nueva Escuela de Arte, septiembre de 1928, Chile. Ejercicios de composición y color del "Primer año de prueba". Fuente: Archivo Pedro Álvarez Caselli.

5. Ejercicios de tipografía Funcionalista y Composición Tridimensional de 1934, enseñados en la Escuela de Artes Aplicadas de la Universidad de Chile desde la reforma de 1928. Fuente: Archivo David F. Maulen.
6. Ejercicios de tipografía Funcionalista y Composición Tridimensional de 1934, enseñados en la Escuela de Artes Aplicadas de la Universidad de Chile desde la reforma de 1928. Fuente: Archivo David F. Maulen.
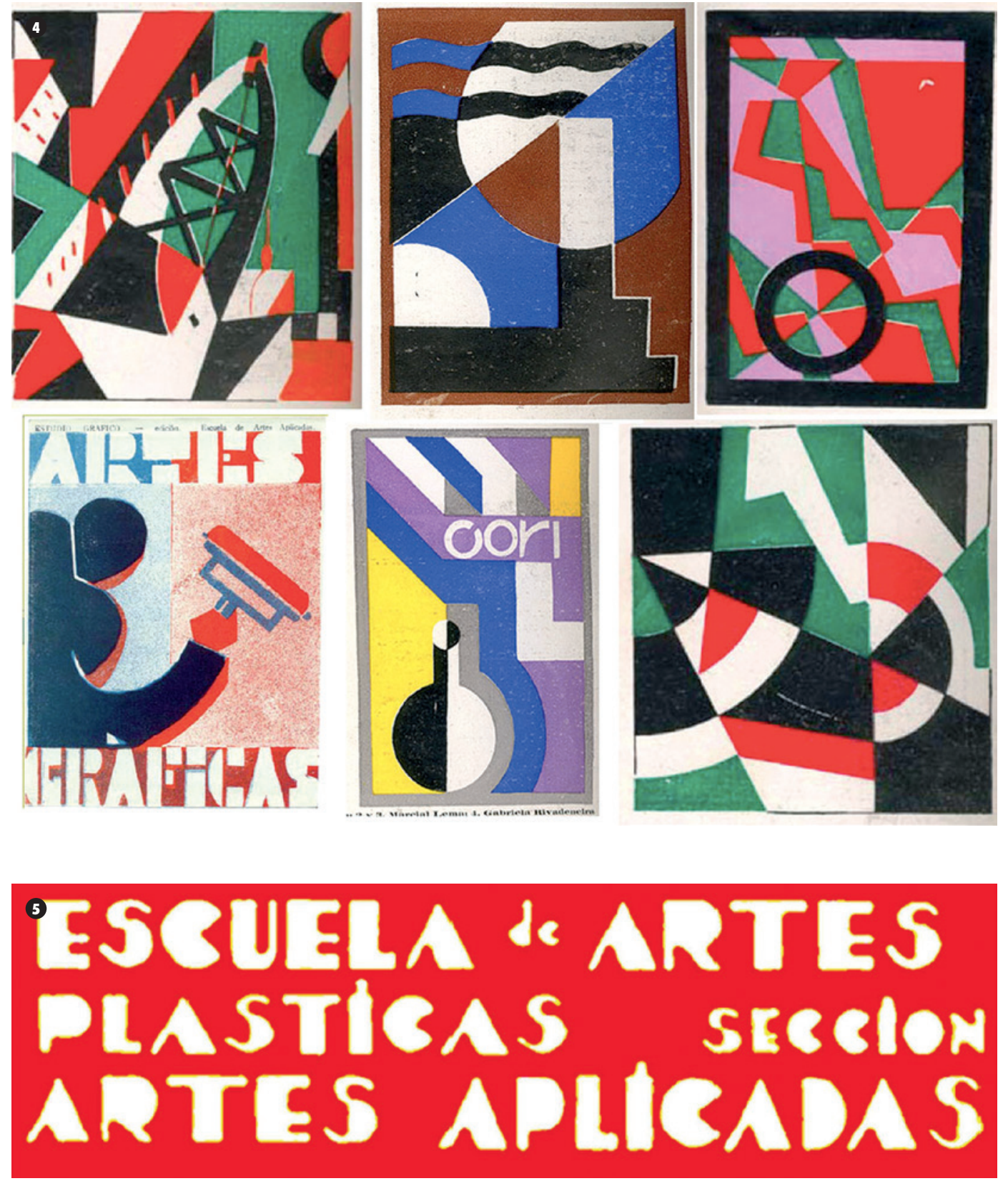

En Chile pasaron cuarenta años antes que una nueva "Reforma Universitaria" implementara un "primer año común" en la "Escuela de Arte" o Facultad de Bellas Artes, con las asignaturas de "forma y espacio" y "forma y color" (basada en la "Gestalt" de Rudolf Arnheim) (Poblete y cols., 1972). Sorprendentemente, los gestores de esta nueva reforma creían ser los primeros en aplicar esta metodología desconociendo el proceso experimentado en 1928, el cual por su complejidad e influencia posterior, seguirá siendo un tema de revisión pendiente.

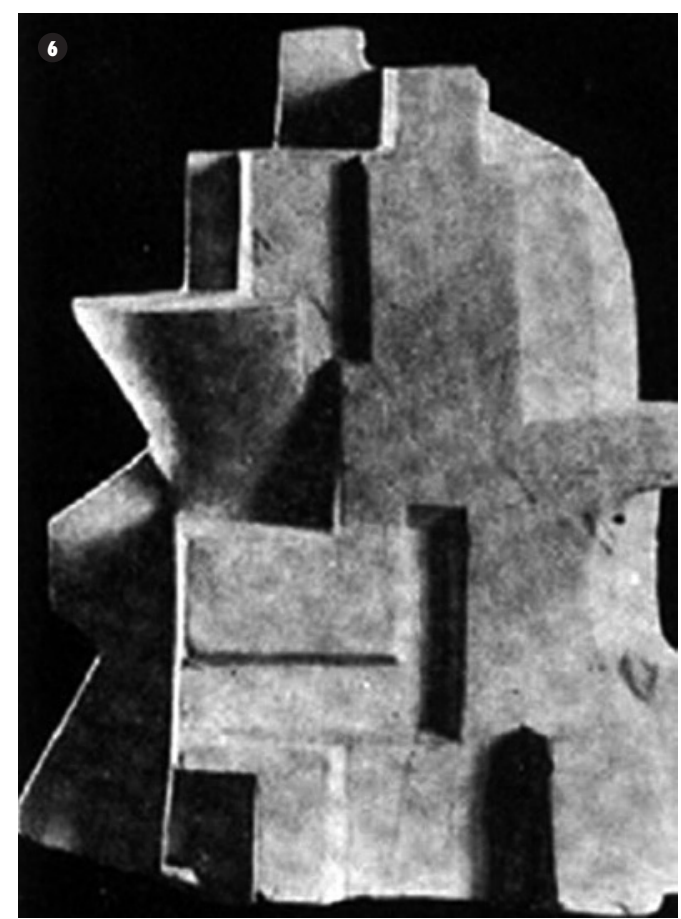

\section{LOS INTENTOS DE RECOMPONER EL MOVIMIENTO, POSTERIORES A LA INTERRUPCIÓN AUTORITARIA DE 1929}

El diseño para el edificio de la Bauhaus en Dessau, obra del propio Walter Gropius, se publicó en la Revista de la Facultad de Arte de la Universidad de Chile en 1934. Por esa época Sergio Larraín García-Moreno mostraba las revistas Bauhaus que había traído de Alemania en 1928, a sus alumnos Jorge Aguirre y Roberto Matta, en la Universidad Católica de Chile (Carrasco, 1987). Matta incluso replicaría comercialmente los muebles de tubería de Marcel Brever. Cerca de 1940 Emilio Duhart, otro estudiante de esta escuela seguiría este impulso renovador de Sergio Larraín, postulando a un programa de estudios con Walter Gropius en la Universidad de Harvard (Montealegre, 1994).

En forma paralela entre 1930 y 1932 el becario chileno Roberto Dávila Carson contacta a Mies van der Rohe para ingresar a la Bauhaus 
7. Proyecto de Escuela de Arte y Arquitectura realizado por Roberto Dávila Carson. Fuente: Revista Moderne Bauformen, 1932
8. Portada Revista ARQuiteCTURA $n^{\circ} 1$ de Waldo Parraguez y Enrique Gebhard, 1935. Incluía el artículo de Walter Gropius "Fundamentos Sociológicos para la Vivienda Mínima". Fotografía de Maximiliano Atria, 2007.

9. Trabajos de la artista Gabriela Rivadeira y de los arquitectos Jaime Dvoredsky (Dvor) y Waldo Parraguez, en 1933 , cercanos a las investigaciones de Lazlo Moholy-Nagy. Fuente: Catálogo de la Exposición Decembristas 1933, y RevISTA ARQUITECTURA n 1 1, 1935. Archivo DIBAM.

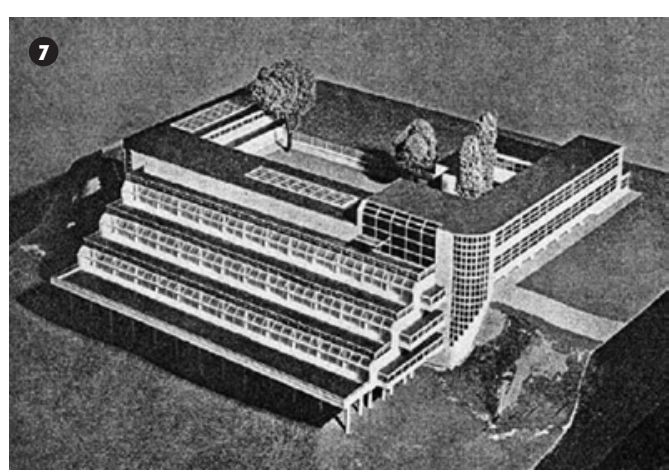

de Dessau, y llega a estudiar con Peter Behrens (maestro de Mies y Gropius), George Vantongerloo y Theo Van Doesburg (influencias constructivistas de la Bauhaus en 1924)

(Chauriye, 2012). Es el único estudiante de Behrens seleccionado para publicar en la revista alemana Moderne Bauformen. Dávila ingresaría como docente a la Universidad de Chile en 1933, tras un movimiento de reforma que creó el curso de "Composición Arquitectónica".

Otros estudiantes reformistas de esa misma universidad, Waldo Parraguez y Enrique Gebhard, publican en su revista ARQUITECTURA de 1935, un artículo de Walter Gropius sobre los fundamentos sociológicos de la vivienda mínima. Parraguez se destacó en la década de 1930 por sus edificios de vivienda social según ideas Bauhaus como la emblemática "Casa 5000", Centro Comunitario de los edificios colectivos del barrio San Eugenio. Anteriormente, en 1933, con Gabriela Rivadeneira [ex alumna del primer año de prueba de 1928] y José Dvoredsky, Parraguez ya había creado un grupo de arte "concreto", Decembristas, con obras similares a las de Laszlo Moholy Nagy, autor del libro "La Nueva Visión". Para entonces Moholy-Nagy había contactado a György Kepes en Berlín. En general, este vínculo también será importante para la transmisión de las ideas de la Bauhaus en América.

En 1942 Emilio Duhart cursó un magíster en la Universidad de Harvard con Walter Gropius, y posteriormente trabajó en su oficina, siendo muy influenciado por sus conceptos de planificación (urbanismo) y vivienda social. De forma
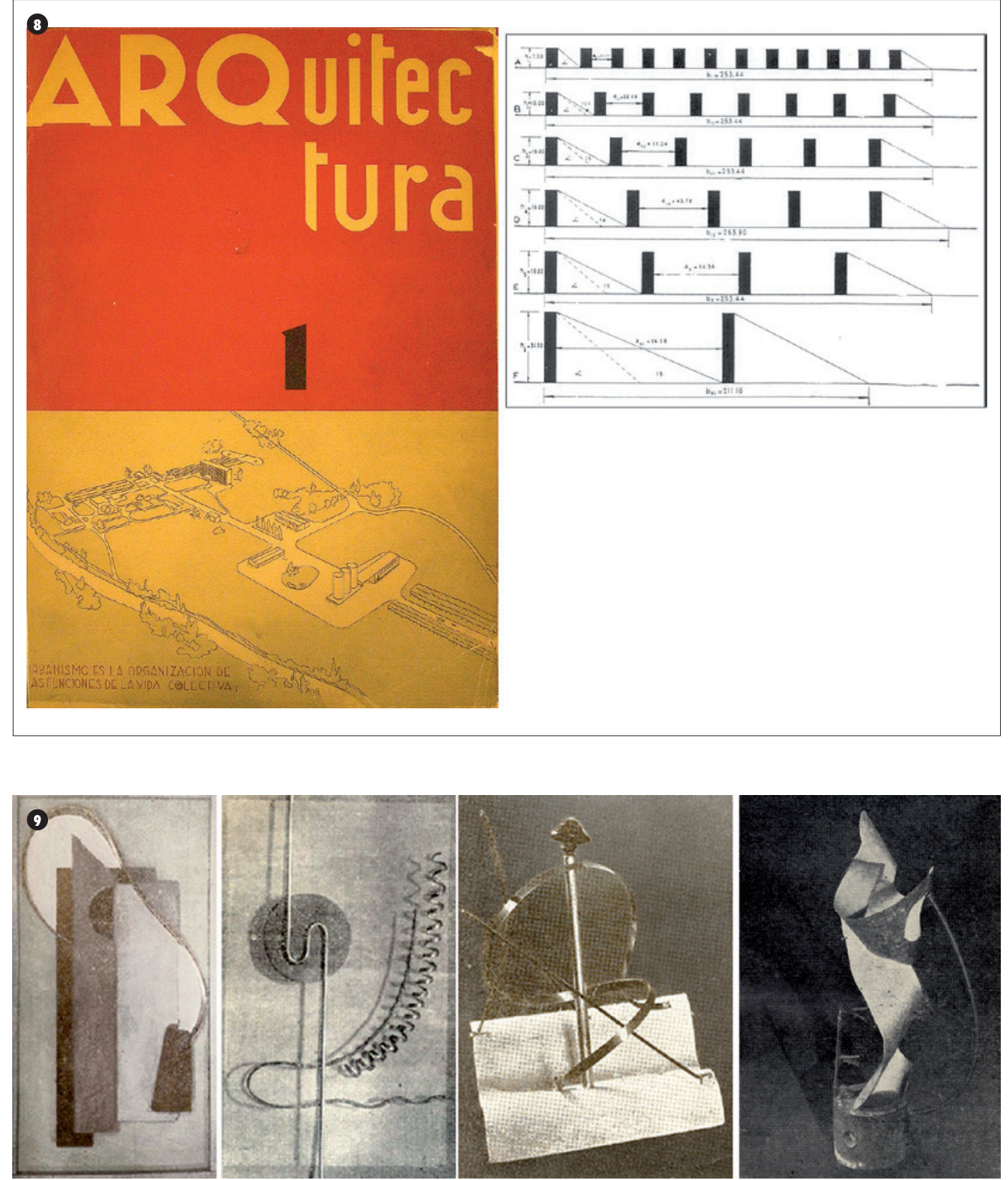

complementaria también asistió a cursos de György Kepes y Laszlo Moholy Nagy.

Después de la Segunda Guerra Mundial, los ióvenes técnicos del Cono Sur se unen en un esfuerzo común de reciprocidad que duraría más de 15 años, siendo el punto de partida principal el Congreso de 1947 en la Universidad
Nacional de Ingeniería de Lima (Barrenechea y cols., 1999). La presentación del modelo de la Arquitectura Integral de la Universidad de Chile, fue preparada por los dirigentes Jorge Bruno González y Abraham Schapira, con supervisión del ex miembro de la Bauhaus Tibor Weiner (Shapira \& González, 1947), y la exposición de planteamientos fue realizada por 
10. Casa 5000, Club Obrero en la Población Arauco, de la Caja de la Habitación Popular de Waldo Parraguez. Fuente: REVISTA URBANISMO Y ARQUITECTURA $n^{\circ} 12,1941$ Archivo Hemeroteca Biblioteca Nacional de Chile.

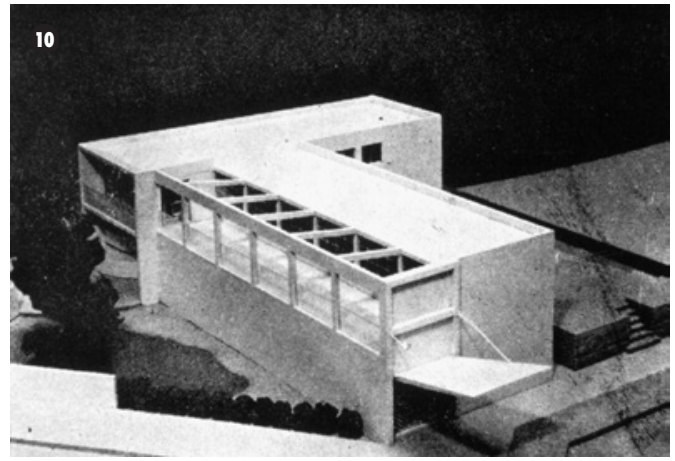

el profesor de Bio Arquitectura, doctor José Garciatello. Complementariamente, desde 1939 Tibor Weiner diseña importantes edificios en las ciudades de Chillán, Concepción y Santiago. Desde el mismo año, tras un breve paso por Chile, otro ex alumno del Bauhaus Paul Linder hará lo propio en Perú, y Marcel Brever en Mar del Plata en 1947.

\section{CONSOLIDACIÓN DE LA BAUHAUS EN LA ENSEÑANZA DE LA ARQUITECTURA CHILENA DESPUÉS DE 1945}

En 1939 el arquitecto húngaro Tibor Weiner -ex asistente del segundo director Bauhaus Hannes Meyer- llega a Chile como refugiado. Weiner se quedó nueve años y fue fundamental en la formulación del Nuevo Plan de Estudios de la Escuela de Arquitectura de la Universidad de Chile desde 1946 (Talesnik, 2013), el "Arquitecto Integral". Este tenía tres ejes principales: el ser Humano, la Naturaleza y la Materia (Maulen, 2006), los cuales se dividían en dos años del ciclo de análisis, y tres años del ciclo de síntesis. Weiner impartió el ramo de Análisis Arquitectural y un taller sobre trazados reguladores. Sus estudiantes publicaban el boletín "Nueva Visión", con textos de Hannes Meyer en México, y con anterioridad habían organizado el Grupo Plástico de Arquitectura, a través del cual difundían los resultados de esta nueva enseñanza. Varios de ellos -Miguel Lawner, Sergio González, Osvaldo Cáceres, Abraham Schapira, Julio Mardones, Raquel Eskenazi, Ricardo Tapia Chuaqui y Ana María Barrenechea, entre otros-, continuaron como

11. Mercado de Concepción, proyecto de Tibor Weiner y Ricardo Müller, 1940. Fuente: Libro de Oro de la ciudad de Concepción, 1950.

12. Credencial de identificación de Hannes Meyer otorgada por el Consulado de México en Francia. Meyer, segundo director de la Bauhaus, trabajó como arquitecto y urbanista en importantes instituciones de los gobiernos de Lázaro Cárdenas y Manuel Avila, desde 1939. Hasta 1939 colaboró con el Taller de Gráfica Popular (TGP). Fuente: Gorelik, Adrián; y Liernur, Francisco. La sombra de la vanguardia, Proyecto Editorial, Buenos Aires, 1993.

13. Borrador presentación reforma Universidad de Chile 1946, al Congreso UNI Lima, 1947. Abraham Schapira, Jorge Bruno González. Con supervisión de Tibor Weiner.

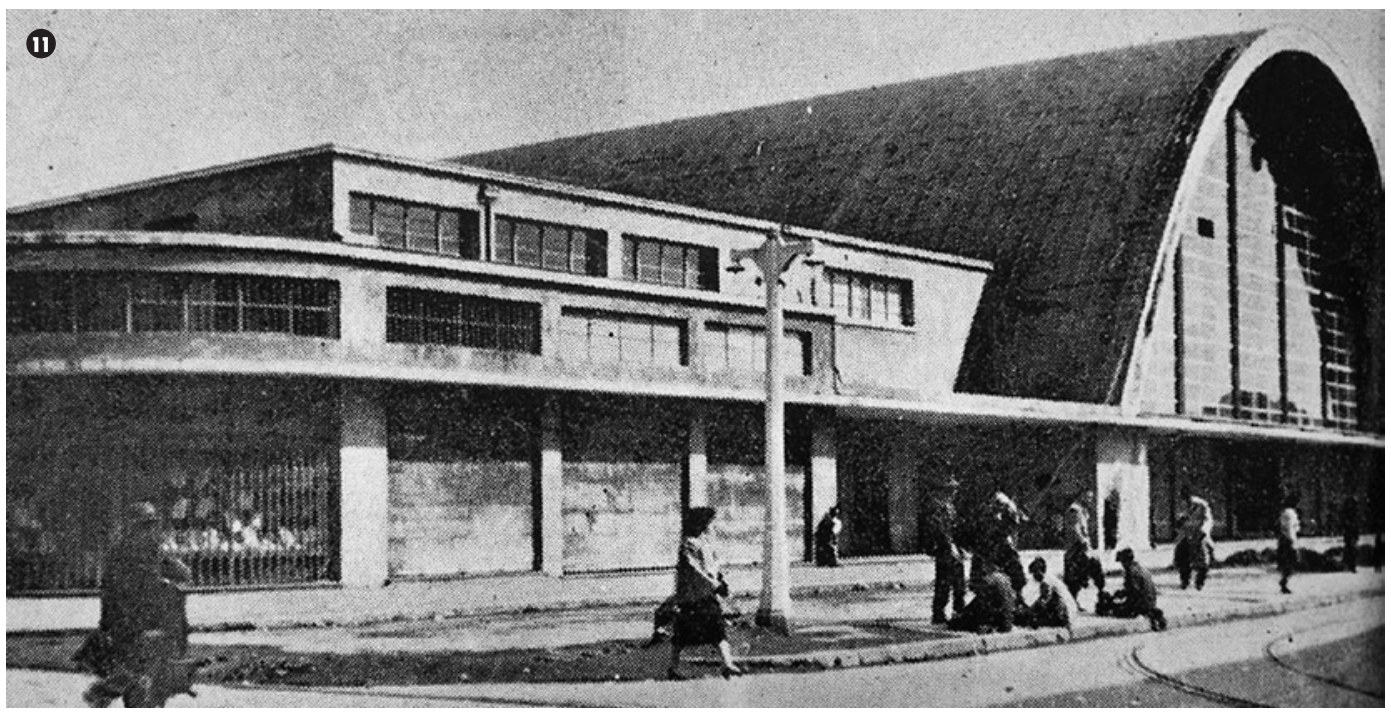

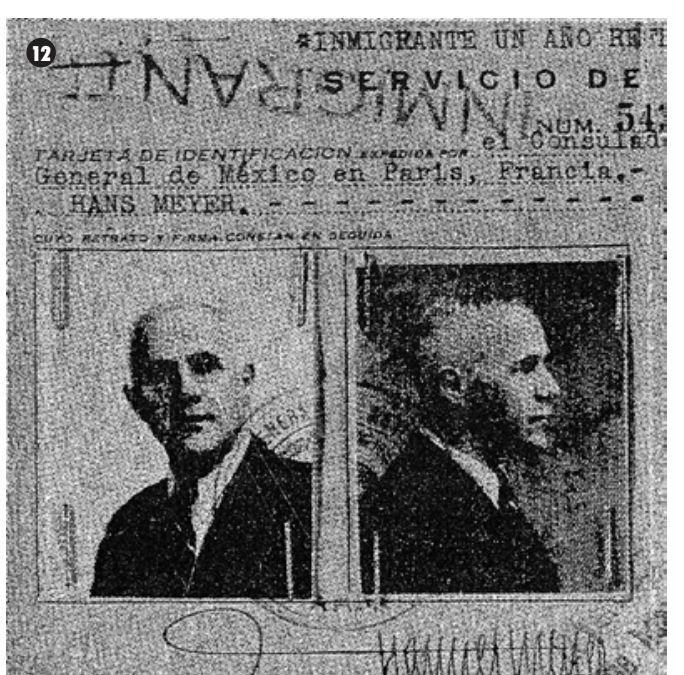

profesores de su Escuela siguiendo estas metodologías que fomentaban el trabajo en equipo, y exploraban la equivalencia de la biología y la anatomía con la arquitectura y el urbanismo. De esta manera, se replanteaban el diseño de la ciudad como un organismo vivo, organizando un trabajo multidisciplinario que establecía una relación dialéctica con el contexto social en el que se aplicaba (Barrenechea y cols., 1999).

Una medida de los alcances de todas estas iniciativas se dio cuando en 1948 Paul Lester

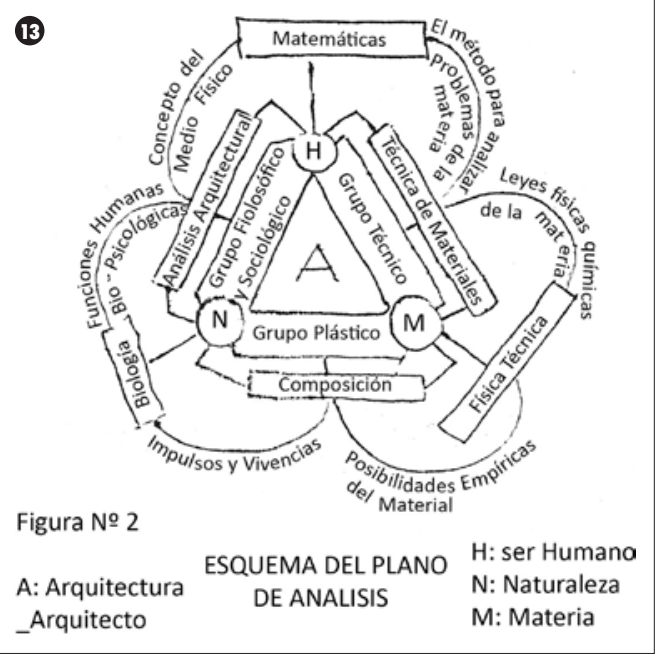

Wiener -cercano a Walter Gropius- visitó Chile y dijo que a su parecer la asignatura de plástica (primer año) era superior a la de la propia Bauhaus. Al mismo tiempo, Ricardo Tapia Chuaqui le escribe a Tibor Weiner sobre las conversaciones con la Escuela de Artes Aplicadas (creada por Carlos Isamitt en 1928) para generar un tipo de Bauhaus con la Escuela de Arquitectura ${ }^{[2]}$.

[2] Carta de Ricardo Tapia Chuaqui a Tibor Weiner en 1948, archivo Miguel Lawner. 
14. Borrador documento de presentación reforma Universidad de Chile, 1946, para Congreso UNI Lima, 1947. Abraham Schapira, Jorge Bruno González. Con supervisión de Tibor Weiner.

15. Cálculo básico de la molécula básica de habitar. Superposición de 4 láminas realizadas por el estudiante Ricardo Tapia Chuaqui para el curso de Análisis Arquitectural del profesor Tibor Weiner, en la Universidad de Chile, 1946. Fuente: Ricardo Tapia Chuaqui.
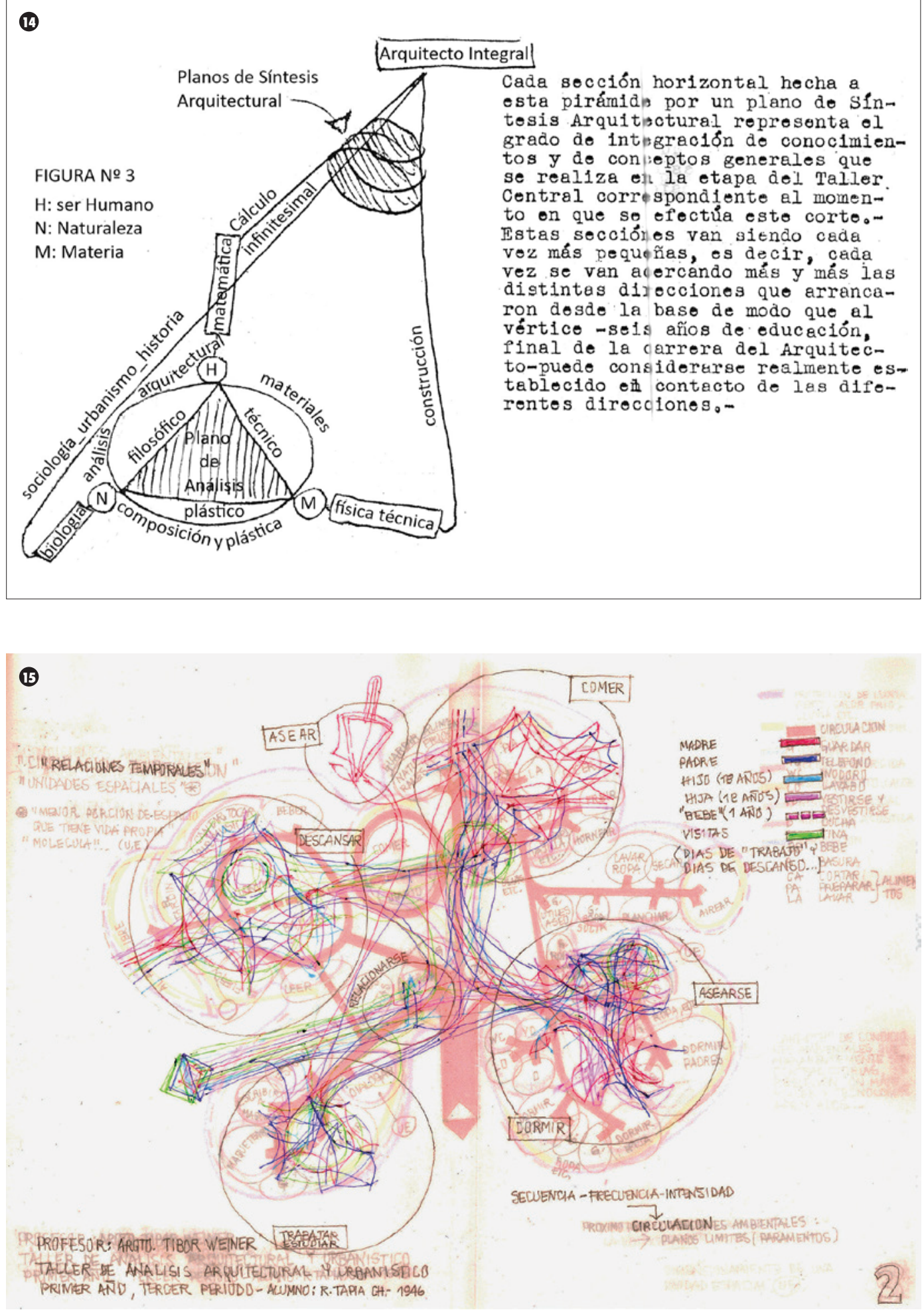

Para ejemplificar la conciencia que existía entre los técnicos chilenos del significado de la Bauhaus es posible revisar un artículo de 1947 escrito por Guillermo Ulriksen en la revista ARquitectura y CONSTRUCCIÓN, EN ChILE, en el cual describe detalladamente sus distintas etapas y orientaciones bajo la dirección de Walter Gropius, Hannes Meyer y Mies van der Rohe. En ese mismo año, encabezados por Fernando Belaúnde Terry, arquitectos de Santiago de Chile, Buenos Aires, Tucumán, Montevideo y Ciudad de México que apoyaban este movimiento se reúnen en el recién creado Departamento de Arquitectura de la UNI de Lima, que luego visitarían figuras como José Luis Sert, cofundador del movimiento promotor del Bauhaus en Barcelona (Gatpac), además del emblemático Walter Gropius y Josef Albers. Esta reunión de 1947 significó el comienzo de un proyecto regional entre los técnicos que querían materializar los principios de la modernidad a través de una "Arquitectura Integral" en Latinoamérica.

Es necesario mencionar además que sin vincularse a la docencia, pero sí desarrollando diseño contemporáneo, otro fundador del Gatpac de Sert, Germán Rodríguez Arias, siguió varios años trabajando en Chile desde su arribo como refugiado en 1939.

En 1949 Sergio Larraín implementa la reforma de la Escuela de Arquitectura de la Universidad Católica de Chile, influenciado fuertemente por Walter Gropius. Una de las innovaciones de esta nueva docencia fue el curso de composición arquitectónica de Alberto Cruz (Baeza, 2009). Esta asignatura tenía cercanía con el "Vorkurs" creado por Josef Albers en la Bauhaus de 1926. En 1952 Emilio Duhart-socio de Sergio Larraínconsiguió que el mismo Josef Albers viajara a Chile.

El "Vorkurs" que realiza Josef Albers en 1953 es muy importante, aun cuando existía el antecedente interrumpido de Carlos Isamitt en 1928, y el de su alumno Ventura Galván en la Escuela de Arquitectura de la Universidad de Chile desde 1946. El taller de Josef Albers fue determinante en alumnos como Alberto 
16. Primera exposición del Grupo Plástico de Arquitectura, 1947, Estudiantes de Arquitectura de la Universidad de Chile. Fuente: Ana María Barrenechea y Miguel Lawner.

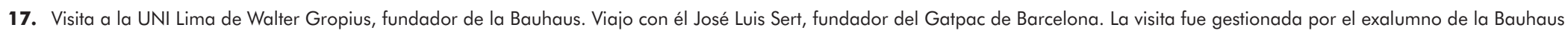
de Weimar Paul Linder, quien participaba del plan de reforma encabezado por Fernando Belaúnde Terry en la Universidad Nacional de Ingeniería desde 1947. Fuente: Medina Warmburg, Joaquín. "Paul Linder: arquitecto, crítico, educador" RA Revista De Arquitectura, Navarra, 2004.

18. Escultura de Gerhard Marcks donada por el Gobierno de Alemania a Chile en 1951. Instalada en el Cerro Santa Lucía, con la firma característica del artista, exprofesor de la Bauhaus de Weimar Fuente: Fotografía de Gonzalo Vargas Malinowski, 2014.
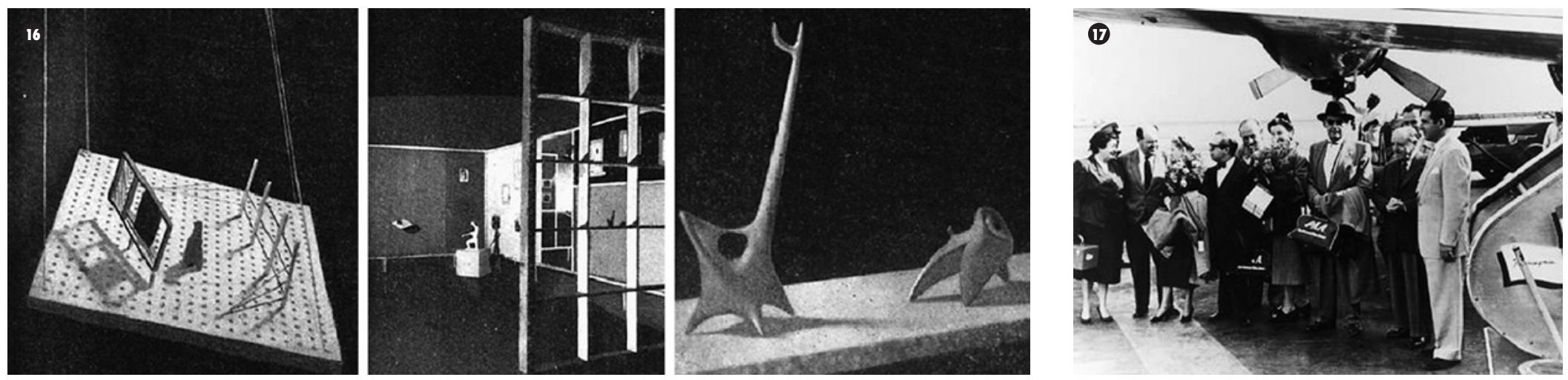

Piwonka, Bernardo Trumper, Julián Larraín, León Rodríguez y Alberto Montealegre, entre otros.

Posteriormente, desde la Universidad de Yale, Albers continuó apoyando a la Universidad Católica de Chile, particularmente en la creación de la Escuela de Arte de 1959, enviando a su mejor discípulo Sewell Sillman a dictar un "Vorkurs". Uno de los alumnos chilenos de Sillman, Eduardo Vilches, perfeccionaría esta enseñanza con el mismo Sillman, gracias a una beca en Estados Unidos. A la vuelta a Chile, Vilches continuó impartiendo esta metodología del "Vorkurs" como docente de Arte de la Universidad Católica de Santiago (Palmarola, 2007). En paralelo, Alberto Piwonka desde 1955 había reformulado el curso de Composición en la enseñanza del Diseño según estas ideas en la misma Facultad.

\section{OTROS CAMINOS DE INTERCAMBIO DIRECTO ENTRE ARTE, ARQUITECTURA Y DISEÑO}

Como representante del espíritu de su época la Bauhaus integraba distintas expresiones de generación de significado del ser humano diluyendo las categorías de la modernidad del siglo XIX entre arte, arquitectura y diseño. Fue así que las dos principales Escuelas de Arquitectura en Chile reflejarán reinterpretaciones de las dos principales tendencias del referente aludido, pero además que los caminos de intercambio y reinterpretación no se agotarán ahí.

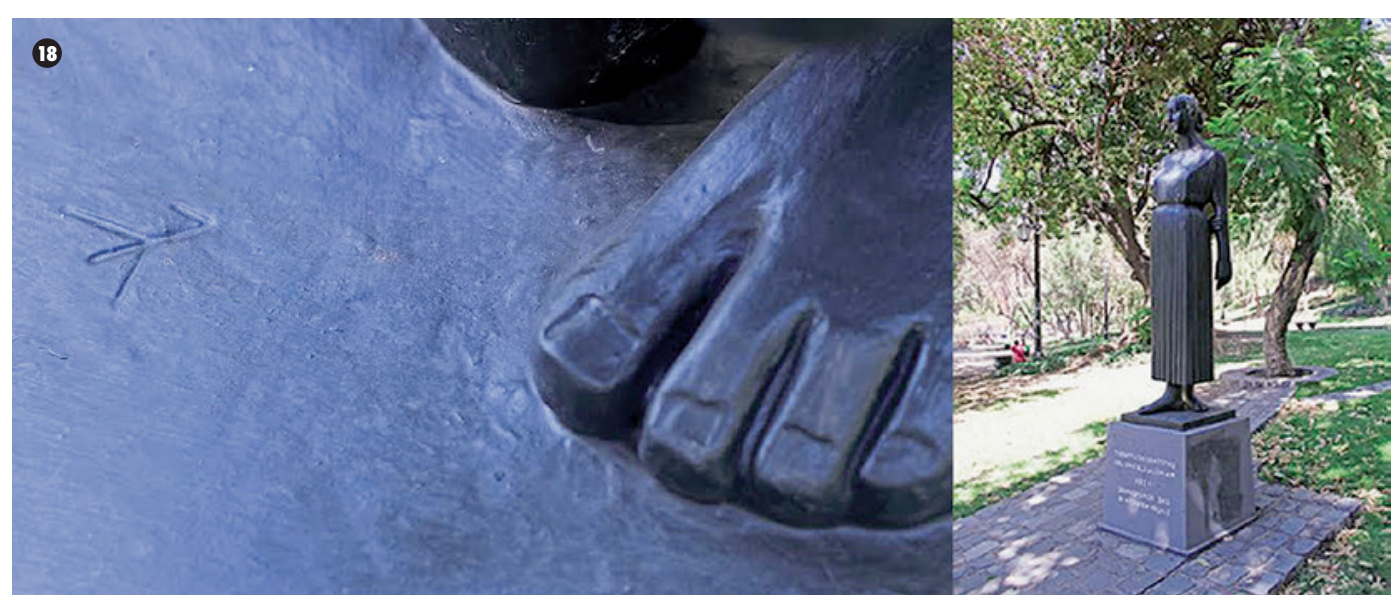

En la época de este impulso por consolidar una modernidad utópica en Sudamérica, en 1951 Alemania regaló a Santiago de Chile una escultura de Gerhard Marks, la cual se instaló en el cerro Santa Lucía. Marcks fue miembro fundador de la Bauhaus, además de escultor fue profesor de cerámica, diseñador industrial, e investigador de la cerámica tradicional de Weimar. Su obra es una síntesis de estas prácticas que colocaban al artesano en el rol protagónico de un diseñador incorporado a los desafíos de la época.

Después de la Segunda Guerra Mundial el impulso inicial de la Bauhaus no se perdió y en 1953 en Ulm (Alemania) se creó la Nueva Bauhaus. Diseñada y dirigida hasta 1959 por el exalumno Bauhaus Max Bill que en 1965 colabora para un Centro Comunitario en Valparaíso conducido como Villa de
"Autoconstrucción" por sus ex alumnos en Ulm: Cornelia Koch, y el arquitecto chileno Eduardo Vargas, quienes indirectamente aproximaron en la realidad local la idea de trabajo en equipo experimentada originalmente por Hannes Meyer y Walter Gropius.

La Facultad de Arquitectura de la Universidad de Chile de Santiago había interrumpido drásticamente el modelo de la Arquitectura Integral de 1945/1946, pero luego de la reforma de 1968 sería la Facultad de Arte y Tecnología de la Universidad de Chile de Valparaíso la que continuó con la anterior orientación, siendo luego elegido Vicedecano el exayudante del curso de Bio Arquitectura de Santiago, Carlos Martínez Corbella. Sería esta sede, entonces, la que luego de la experiencia de la Villa Berlín, invitaría a Eduardo Vargas a impartir el "Taller Vargas" (Vargras Koch, 2007). 
19. En 1953 en la ciudad de Ulm, Alemania empieza el proyecto de la "nueva Bauhaus", la HfG Ulm, Max Bill diseña el edificio y será su director hasta 1959. En 1953 Bill, exalumno de la Bauhaus en el período de cambio de Director de Walter Gropius a Hannes Meyer, visitó Brasil. Luego Bill escribió un artículo para Architectural Review publicado en 1954: "Reporte sobre Brasil". En la foto Bill aparece visitando el Ministerio de Educación diseñado por Lucio Costa y Oscar Niemeyer, con ayuda de Le Corbusier entre 1936 y 1945. Fuente: García, María Amalia. "Tensiones entre tradición e innovación" en Cocinnitas, Vol 1, Río de Janeiro, 2010.
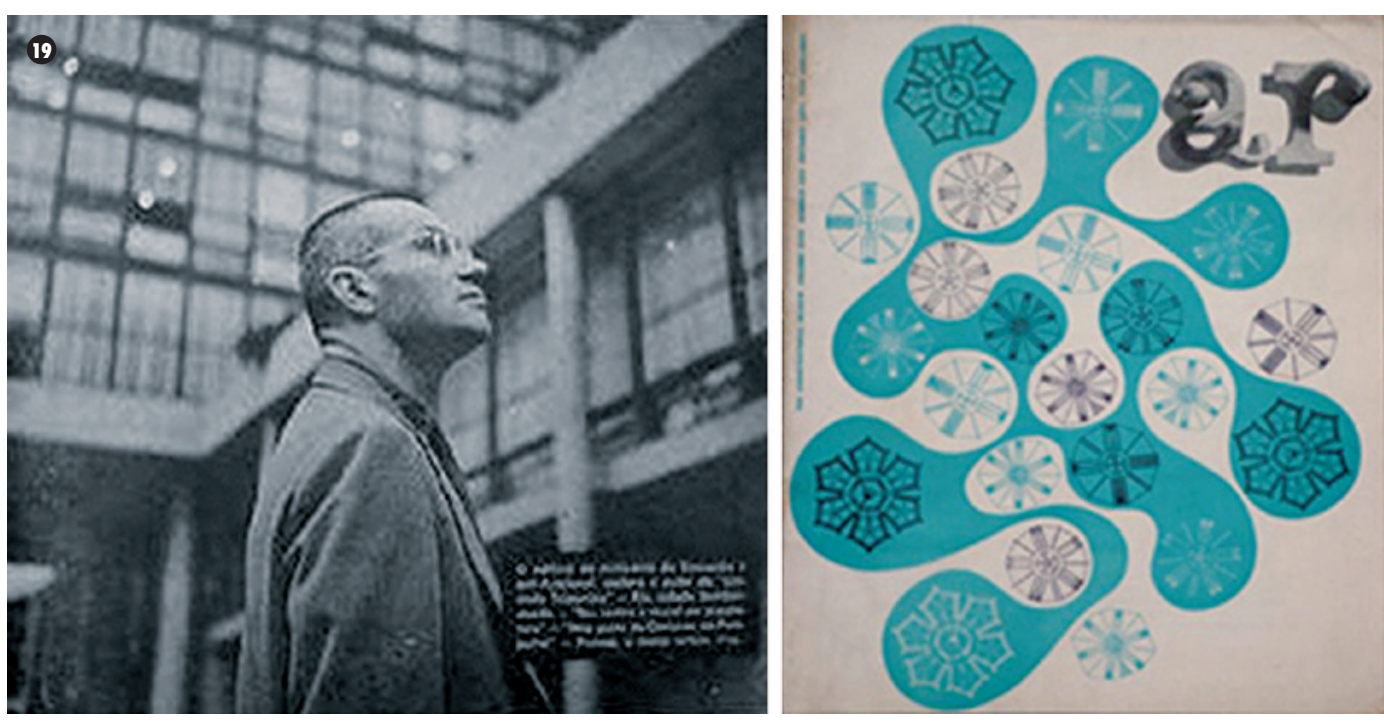

Los planteamientos radicales de la HfG Ulm provocaron su cierre en 1968, y gracias a la Organización Internacional del Trabajo es contratado en la Corporación de Fomento a la Producción (CORFO) Chile uno de sus profesores, Gui Bonsiepe, quien dirigiría su Instituto Tecnológico entre 1971 y 1973, con dos miembros más de la HfG Ulm: Werner Zemp y Michael Weiss (Palmarola, 2003). Este último residirá en Chile hasta 1983 colaborando en el Departamento de Diseño de la Universidad de Chile y en la fundación del Instituto Superior ARCIS.

Un año antes de su partida de Chile en septiembre de 1973, Bonsiepe recibió el encargo de generar dos planes de estudios, los cuales fueron implementados en quinto año de ingeniería de la Universidad Católica, y principalmente en la Facultad de Arte Tecnología de la Universidad de Chile en Valparaíso por intermedio del exmiembro del grupo Plástico de Arquitectura, Sandalio Valdebenito ${ }^{[3]}$.

Por otro lado, desde una perspectiva más subjetiva de lo que fue la herencia de la
Bauhaus, en 1967 uno de los fundadores del Bauhaus de Chicago de 1938 György Kepes creó el Centro de Investigaciones Visuales Avanzadas (CAVS) en el Instituto Tecnológico de Massachusetts. La colaboración de este organismo con artistas, científicos y técnicos chilenos se inició en el número 2 de su revista LEONARDO, publicando el trabajo del ingeniero Carlos Martinoya y el cristalógrafo Nahum Jöel (Maulen, 2013). A continuación, en el año 1969 harían residencias los artistas Virginia Huneeus y Carlos Ortúzar. Ambos a la fecha habían desarrollado arte integrado a la arquitectura. Poco tiempo después también se convierte en alumno de Kepes otro chileno, el ingeniero y artista Alejandro Siña, el cual había sido alumno de Carlos Ortúzar (Maulen, 2002).

Las proyecciones del cruce de disciplinas característico de la Bauhaus siguió su curso en los intercambios entre algunos de sus miembros con creadores chilenos. Por ejemplo, Bernardo Trumper estudió arquitectura en el momento en que se implementó el plan de estudios donde Tibor Weiner tuvo una injerencia decisiva. Años después Trumper asistió al
"Vorkurs" de Josef Albers en la Universidad de Yale, pero su mayor desarrollo no se identifica con la arquitectura ni el diseño concebido tradicionalmente, sino que en otro sentido, en el campo del diseño teatral y la iluminación. Estas dinámicas de reinterpretación e intercambio se podrían ejemplificar de manera inversa con la experiencia del arquitecto chileno Christian Jensen, el cual fue discípulo de Albert Mentzel -alias Flocon- destacado profesor de escenografía del Bauhaus. Jensen estudió con este maestro entre los años 1974 y 1980 en la Escuela de Paris Belleville $e^{[4]}$.

La proyección final de estas reorientaciones de objetivos, continuaron en casos tan destacados como la aplicación de la metodología del Ballet Tríadico de Oskar Schlemmer, en la obra de teatro Santiago Bauhaus (1987), del dramaturgo y director teatral chileno Ramón Griffero (2006), continuador en una nueva época de las resignificaciones que los objetivos de la Bauhaus original tendrían para los creadores locales.

NOTA: a diferencia de los exmiembros del Bauhaus Tibor Weiner, Paul Linder o Joseph Albers, existió un diseñador proveniente de esta Escuela que fallece en Chile 1938 sin que sus planteamientos tuvieran repercusiones en las transformaciones educativas, fue el diseñador de muebles Gunter Hirschel Protsch, de quien es necesario dejar referencia. Agradecemos esta observación a la profesora Magdalena Droste.

\section{BIBLIOGRAFÍA}

Barrenechea, A. M. et al. (1999). A 53 años de la reforma de la Escuela de Arquitectura de la Universidad de Chile. Ínédito.

Carrasco, E. (1987). Matta. Conversaciones. Santiago de Chile: Interamericana.

Chauriye, R. (2012). "Arquitectura moderna en Chile. El caso de Roberto Dávila Carson". En

[3] Testimonio de Rodrigo Walker, discípulo y exasistente de Gui Bonsiepe en Chile. Actividad Little Talks, exposición Clip Stamp Fold, Centro Cultural Gabriela Mistral, Santiago de Chile: agosto 2013

[4] Entrevista con el autor, febrero 2014 
Arquiteturarevista, Vol 8, № 2, pp. 148 a 154 Unisinos. Revisión on line enero 11, 2014.

Griffero, R. (2006). Poéticas de Espacio Escénico Herbert Jonckers - Chile 1981/1996. Santiago de Chile; Ediciones Frontera Sur.

Maulen, D. (2002). Virginia Huneeus, síntesis 1934 a 2002. Santiago de Chile: Catálogo exposición retrospectiva Museo Nacional de Bellas Artes

Maulen, D. (2005). Vernacularidad y Funcionalismo. La Nueva Escuela de Arte, Chile 1928. Inédito: Informe de investigación Fondo de Desarrollo de la Cultura y las Artes.

Maulen, D. (2006). "Inclusión y exclusión del espacio del espacio urbano y social. La Reforma de la Escuela de Arquitectura de la Universidad de Chile, 1933, 1945, 1964". Revista de Arquitectura, № 14. Santiago de Chile: Facultad de Arquitectura y Urbanismo Universidad de Chile.

Maulen, D. (2013). Precursores del arte cinético en Chile, en Revista on line Scanner Cultural $N^{\circ}$ 161, Santiago de Chile.http://www.escaner.cl/ pdf/Precursores_del_Arte_Cinetico_en_Chile_por_ David_F._Maulen_De_los_Reyes.pdf

Molina Baeza, C. (2009). "El proyecto como lugar de síntesis de ideas: Los colegios del Verbo Divino
(1948-1975) y San Ignacio El Bosque (19581972) en Santiago de Chile", Presentación al $8^{\text {vo }}$ Seminario de patrimonio moderno do.co.mo.mo. Brasil. Revisión: enero 11, 2014. http://www. docomomo.org.br/seminario\%208\%20pdfs/104. pdf

Montealege, A. (1994). Emilio Duhart arquitecto. Santiago Ed. Pontifica Universidad Católica de Chile.

Palmarola Sagredo, H. (2003). "Productos y Socialismo: Diseño Industrial Estatal en Chile", en C. Rolle, 1973 - La vida cotidiana de un año crucial, pp. 225 a 295. Santiago de Chile: Editorial Planeta.

Palmarola Sagredo, H. (2007). Cartografía del curso preliminar. Josef Albers y Chile, Albers viajes por Latinoamérica. Madrid: Museo Nacional Centro de Arte Reina Sofía.

Poblete, G. et al. (1972). Informe primer año común, forma y color, forma y espacio, expresión gráfica. Inédito, documento de trabajo Facultad de Bellas Artes Universidad de Chile.

Reyes Jedlicki, L. (2005). Movimientos de educadores y construcción de política educacional en Chile: 1921-1932 y 1977-1994. Tesis doctorado Universidad de Chile.
Schapira, A., González, J. B. (con supervisión de Tibor Weiner).(1947). Informe de la Escuela de Arquitectura de la Universidad de Chile preparado para el Congreso Panamericano de Lima 1947, borrador para la revista Arquitectura y Construcción, Chile. Inédito.

Talesnik, D. (2013). A25 Tibor Weiner. Escuela de Arquitectura, Universidad de Chile, Santiago, Chile 1943-1963. Radical Pedagogies, Action, Reaction, Interaction, pabellón incluído en la 14 Bienal de Venecia, curatoría Beatriz Colomina. Revisión Junio 24, 2014. http://radicalpedagogies.com/

Vargas Koch, S. (2007). "Anteproyecto Centro Comunal Valparaíso - Reflexiones sobre las relaciones de Bill, la HfG y América Latina", trabajo de investigación del curso Arquitecturas de América Latina, Magíster Arquitectura Universidad Católica de Chile. Revisión on line enero 11, 2014. www.magisterarq.cl

Wingler, H. (1975). Marcks, Gerhard, Bauhaus, Weimar, Dessau, Berlin. 1919 1933. Barcelona: Gustavo Gili. 